\title{
Stoichiometric analysis of self-maintaining metabolisms
}

\author{
Francisco Montero , Juan Carlos Nuño , Enrique Meléndez-Hevia , Felix Olasagasti , \\ Sara Vázquez, Federico Morán
}

\begin{abstract}
Departamento de Bioquímica y Biologia Molecular I, Universidad Complutense Madrid, Avd. Complutense s/n, 28040 Madrid, Spain Departamento de Matemática Aplicada a los Recursos Naturales, ETSIM, Universidad Politécnica de Madrid, 28040 Madrid, Spain Instituto del Metabolismo Celular, Calle Manuel de Falla, 15, 38208 La Laguna, Tenerife, Spain
\end{abstract}

\begin{abstract}
This paper presents an extension of stoichiometric analysis in systems where the catalytic compounds (enzymes) are also intermediates of the metabolic network (dual property), so they are produced and degraded by the reaction network itself. To take this property into account, we introduce the definition of enzyme-maintaining mode, a set of reactions that produces its own catalyst and can operate at stationary state. Moreover, an enzyme-maintaining mode is defined as elementary with respect to a given reaction if the removal of any of the remaining reactions causes the cessation of any steady state flux through this reference reaction. These concepts are applied to determine the network structure of a simple self-maintaining system.
\end{abstract}

Keywords: Stoichiometric analysis; Self-maintaining systems; Metabolic networks

\section{Introduction}

It was in Granada (Spain), in September 1994, during an international workshop on evolution of metabolism, that Prof. Heinrich evoked our interest in the stoichiometric analysis of metabolic networks, in particular, its application to the study of the urea cycle. Since then, he has always emphasized the importance of this kind of analysis for understanding cell metabolism (Heinrich and Schuster, 1996).

The stoichiometric analysis of metabolic networks has been intensively developed in the last decade (Nuño et al., 1997; Schilling and Palsson, 1998; Schuster et al., 2000; Klamt and Stelling, 2006). This kind of analysis has proved to be a very useful tool for getting functional information about metabolic networks that can also be applied in metabolic engineering (Schuster et al., 1999; Stephanopoulos et al., 1998; Schilling et al., 1999). Under the steady state assumption, the mathematical analysis of the stoichiometric matrix yields a basis of its null space, the enzyme subsets (Pfeiffer et al., 1999), and the elementary modes (EMs; Schuster et al., 1999). On the basis of this information it is possible to define metabolic pathways and cycles, and to detect correlated reactions (for a review see Schuster et al., 2000; Klamt and Stelling, 2006). However, in this approach the enzymes enter the metabolic network as binary operators that can only be turned on and off externally (Covert and Palsson, 2003), which means that the reaction is either working or not, respectively. This is true for most of the metabolic studies. However, in systems where the network itself involves the formation and degradation of the catalysts, each enzyme has a dual property, as a catalyst and as a metabolite. In the situations mentioned above the results must be complemented with additional information that can modify the results of the stoichiometric analysis, as fundamental modes and subsets present in the network. 
To show the major consequences of considering the dual properties of component operators, in this paper we analyze a previously proposed model of a self-maintained system (Olasagasti et al., 2007).

\section{The self-maintained proto-cellular system model}

A model of a self-maintained proto-cellular system has previously been presented by Olasagasti et al. (2007). This represents a closed membrane maintained by an internal set of reactions (a minimal metaboliclike network) following the basic considerations of the autopoietic model (Varela et al., 1974). The system transports external metabolites into the system and self-constructs its components using an external energy source. This model is an energetically coherent system and fulfils the laws of bioenergetics proposed by Skulachev (1992). Fig. 1 shows a schematic view of this cellular system (see the caption of Fig. 1 for details).

The model assumes that there are different types of components: structural $\left(L_{m}\right)$, capable of self-assembling to build a semi-permeable membrane; catalytic $(E)$, which combine both enzyme-like activity and the transport of molecules across a membrane; transducer molecules $(T)$,

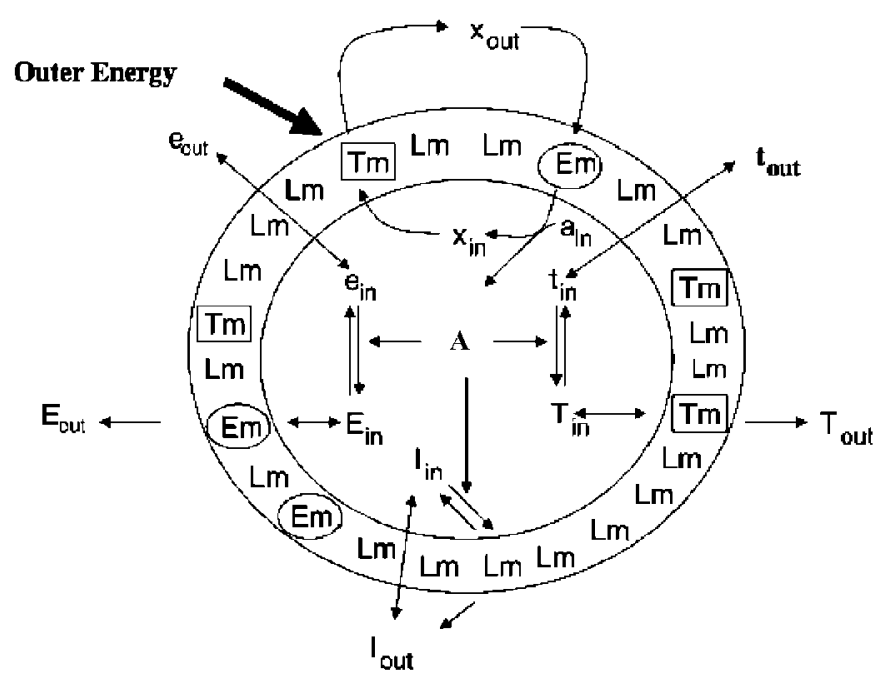

Fig. 1. Representation of the cellular system with the chemical network and membrane components. $t_{\text {out }}, e_{\text {out }}$, and $l_{\text {out }}$ precursors enter through the membrane and once in the system, they use the energy of $A$ (energy-rich molecule) to form $E_{i n}, T_{i n}$, and $L_{m} . E_{i n}$ and $T_{i n}$ molecules can be inserted into the membrane, composed mainly of $L_{m}$, to form $E_{m}$ and $T_{m}$, respectively. The upper cycle represents the energy-transduction mechanism of the system. Outer energy can be used by $T_{m}$ transducers to create a concentration difference of $x$ (a substance that cannot pass through the membrane by free diffusion) between the inner and the outer sides of the membrane. The energy that is released when $x$ passes to the inner side from the outer side can be used by $E_{m}$ (with catalytic activity) to form energy-currency molecules $(A)$ from precursors that are in the system. $E_{m}$ and $T_{m}$ can be broken up to release the external polymers $E_{o u t}$ and $T_{r u t}$, respectively. which can use an external energy source to selectively pump molecules across a membrane; and energy "currency" components $(A)$, which will facilitate the couplings among different reactions (see Fig. 1 caption for a detailed description). All the $T_{m}, E_{m}$, and $L_{m}$ components have the dual property of being both a catalyze and a metabolite.

Table 1 shows the different transformations involved in the self-maintained proto-cellular system metabolism. The enzymatic reactions $\left(v_{12}, v_{13}, v_{14}\right.$, and $\left.v_{15}\right)$ are assumed to follow a Michaelis-Menten mechanism and thus each reaction is split into two steps, mediated by an enzyme-substrate complex. Moreover, it is specified whether each step is reversible or irreversible and whether the metabolites involved in the network are internal or external.

\section{Stoichiometric analysis}

The stoichiometric analysis of the system described in the previous section has been carried out with METATOOL (Pfeiffer et al., 1999; Kamp and Schuster, 2006), yielding the following main results:

(a) Reaction subset (RS): A very important concept that permits the detection of functional units, as well as the reduction of the dimension of the stoichiometric matrix, is the concept that was previously defined as an enzyme subset: a set of enzymes that operate at the steady state at fixed rate proportions (Pfeiffer et al., 1999). This concept can be directly extended to describe metabolic systems formed by reactions that are not enzymatically catalyzed. In these cases, the term $R S$ is more appropriate.

The RSs of the self-maintaining system are described in Table 2. The first three RSs imply a mass balance of monomers and polymers of each species. RSs $5-10$ are single reactions that are not linked stoichiometrically to any other. Subset 4 corresponds to the stoichiometric coupling between the energy-consuming output flux of $\mathbf{x}$ and the re-input of $\mathbf{x}$ with a net production of A. It must be emphasized that, although the net mass balance of enzymes $T_{m}$ and $E_{m}$ is zero, this subset can work only with non-null concentrations of both enzymes.

(b) Convex basis and EMs: The dimension of the kernel of the stoichiometric matrix is 6 . Since the number of EMs is equal to the dimension of the kernel for this system, a convex basis is that formed by these EMs, as shown in Table 2. The general solution, expressed as a vector of the rates of the RSs $\left(R S_{i}\right)$, can be written as a convex combination of the normalized rate vectors corresponding to 
Table 1

Intermediates and reactions for the network depicted in Fig. 1

\begin{tabular}{|c|c|c|c|}
\hline Abbreviation & Description & Reaction equations & Character \\
\hline$v_{1}$ & Input/output of $l$ & $l_{o u t}=l_{i n}$ & Reversible \\
\hline$v_{2}$ & Input/output of $e$ & $e_{o u t}=e_{i n}$ & Reversible \\
\hline$v_{3}$ & Input/output of $t$ & $t_{\text {out }}=t_{\text {in }}$ & Reversible \\
\hline$v_{4}$ & Synthesis of $L_{m}$ & $l_{i n}+A=L_{m}+a$ & Irreversible \\
\hline$v_{5}$ & Synthesis of $E_{\text {in }}$ & $e_{i n}+A=E_{i n}+a$ & Irreversible \\
\hline$v_{6}$ & Synthesis of $T_{i n}$ & $t_{i n}+A=T_{i n}+a$ & Irreversible \\
\hline$v_{7}$ & Degradation of $L_{m}$ & $L_{m}=l_{i n}$ & Irreversible \\
\hline$v_{8}$ & Degradation of $E_{i n}$ & $E_{i n}=e_{i n}$ & Irreversible \\
\hline$v_{9}$ & Degradation of $T_{\text {in }}$ & $T_{i n}=t_{i n}$ & Irreversible \\
\hline$v_{10}$ & Formation of $E_{m}$ & $E_{i n}+L_{m}=E_{m}$ & Reversible \\
\hline$v_{11}$ & Formation of $T_{m}$ & $T_{i n}+L_{m}=T_{m}$ & Reversible \\
\hline$v_{12}$ & Coupling of energy with output of $x$, catalyzed by $T_{m}$ & $x_{i n}+$ energy $+T_{m}=T_{m x}$ & Irreversible \\
\hline$v_{13}$ & & $T_{m x}=T_{m}+x_{o u t}$ & Reversible \\
\hline$v_{14}$ & Coupling of $x$ input with synthesis of $A$, catalyzed by $E_{m}$ & $x_{o u t}+a+E_{m}=E_{m x}+A$ & Reversible \\
\hline$v_{15}$ & & $E_{m x}=E_{m}+x_{i n}$ & Reversible \\
\hline$v_{16}$ & Output of $l$ & $L_{m}=l_{\text {out }}$ & Irreversible \\
\hline$v_{17}$ & Output of $E_{m}$ & $E_{m}=E_{o u t}+L_{m}$ & Irreversible \\
\hline$v_{18}$ & Output of $T_{m}$ & $T_{m}=T_{\text {out }}+L_{m}$ & Irreversible \\
\hline
\end{tabular}

External compounds

Internal intermediates

$L_{i n} e_{i n} t_{i n} A$ a $L_{m} E_{i n} T_{i n} E_{m} T_{m} x_{\text {in }} x_{o u t} T_{m x} E_{m x}$

each EM:

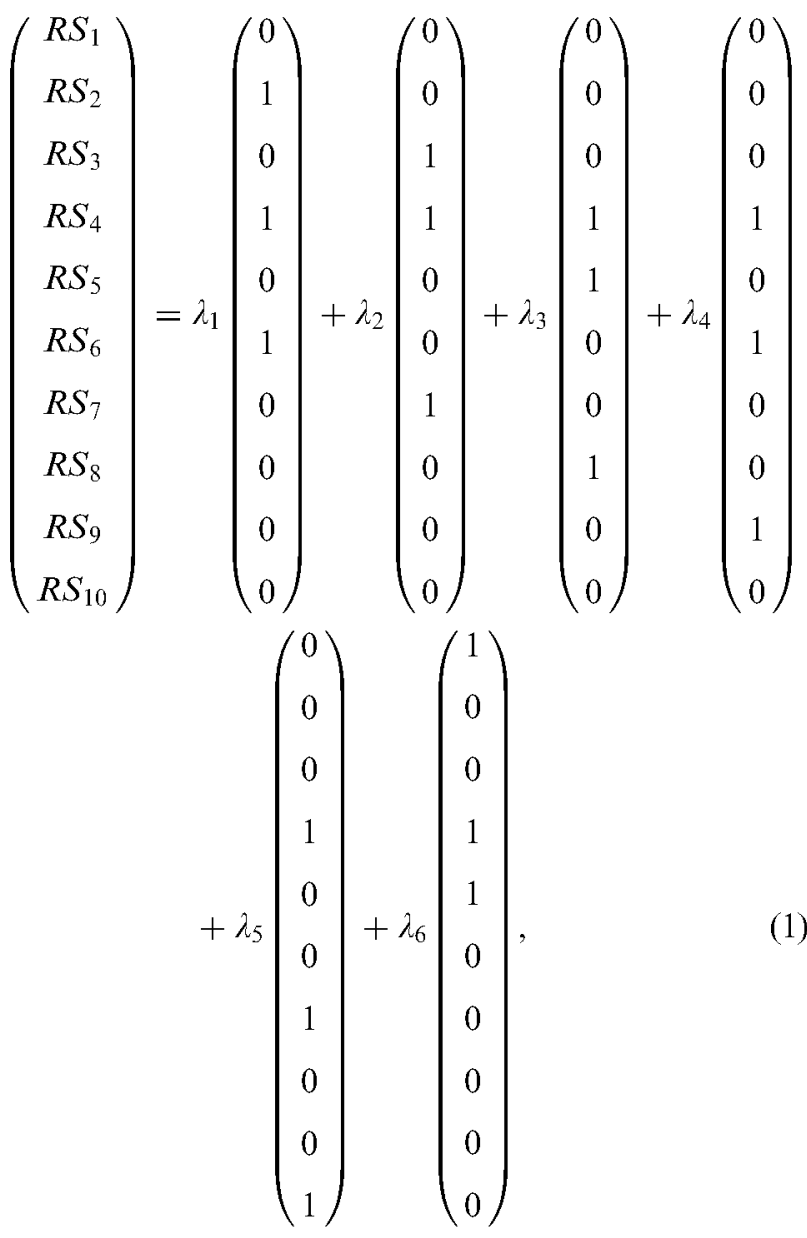

Table 2

Reaction subsets (RS) and elementary modes (EM) for the reaction network depicted in Fig. 1

\begin{tabular}{llll}
\hline RS & Overall reaction & $\begin{array}{l}\text { Participating } \\
\text { reactions and fluxes }\end{array}$ & Character \\
\hline 1 & $L_{m}=l_{\text {in }}$ & $v_{1} v_{16}$ & Irreversible \\
2 & $E_{\text {in }}+e_{\text {out }}=e_{\text {in }}+E_{\text {out }}$ & $v_{2} v_{10} v_{17}$ & Irreversible \\
3 & $T_{\text {in }}+t_{\text {out }}=t_{\text {in }}+T_{\text {out }}$ & $v_{3} v_{11} v_{18}$ & Irreversible \\
4 & $a+$ energy $=A$ & $v_{12} v_{13} v_{14} v_{15}$ & Irreversible \\
5 & $l_{\text {in }}+A=L_{m}+a$ & $v_{4}$ & Irreversible \\
6 & $e_{\text {in }}+A=E_{\text {in }}+a$ & $v_{5}$ & Irreversible \\
7 & $t_{\text {in }}+A=T_{\text {in }}+a$ & $v_{6}$ & Irreversible \\
8 & $L_{m}=l_{\text {in }}$ & $v_{7}$ & Irreversible \\
9 & $E_{\text {in }}=e_{\text {in }}$ & $v_{8}$ & Irreversible \\
10 & $T_{\text {in }}=t_{\text {in }}$ & $v_{9}$ & Irreversible \\
& Overall reaction & $\mathrm{Involved} \mathrm{RS}$ & Character \\
\hline 1 & $e_{\text {out }}+\mathrm{energy}=E_{\text {out }}$ & $R S_{2} R S_{4} R S_{6}$ & Irreversible \\
2 & $t_{\text {out }}+$ energy $=T_{\text {out }}$ & $R S_{3} R S_{4} R S_{7}$ & Irreversible \\
3 & Energy $=$ & $R S_{4} R S_{5} R S_{8}$ & Irreversible \\
4 & Energy $=$ & $R S_{4} R S_{6} R S_{9}$ & Irreversible \\
5 & Energy $=$ & $R S_{4} R S_{7} R S_{10}$ & Irreversible \\
6 & Energy $=$ & $R S_{1} R S_{4} R S_{5}$ & Irreversible \\
\hline
\end{tabular}

where $\lambda_{i} \geqslant 0$, for all $i=1,2, \ldots, 6$ due to the irreversible character of the reactions concerned. The sub index of $\lambda_{i}$ refers to the corresponding EM $\left(E M_{i}\right)$.

Fig. 2 shows a schematic representation of the six EMs. Modes 1 and 2 represent the energetically driven synthesis of $E_{\text {out }}$ and $T_{\text {out }}$, respectively. In both modes, $E_{m}$ and $T_{m}$ participate as catalysts although they are not both internally produced in each of them. This is also the case 

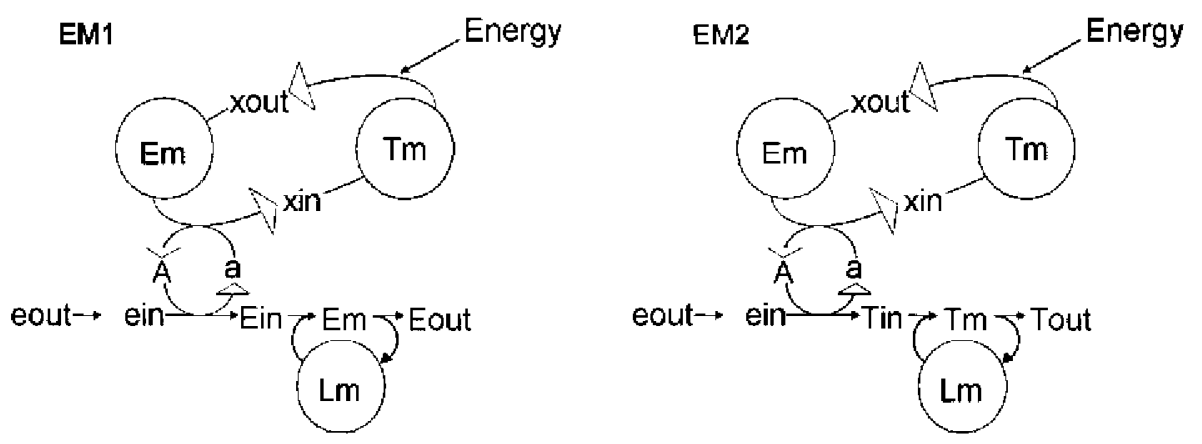

EM3

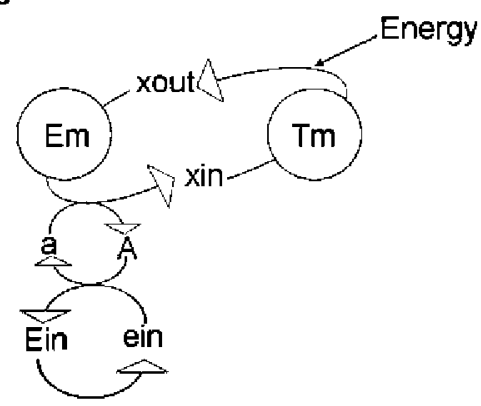

EM4
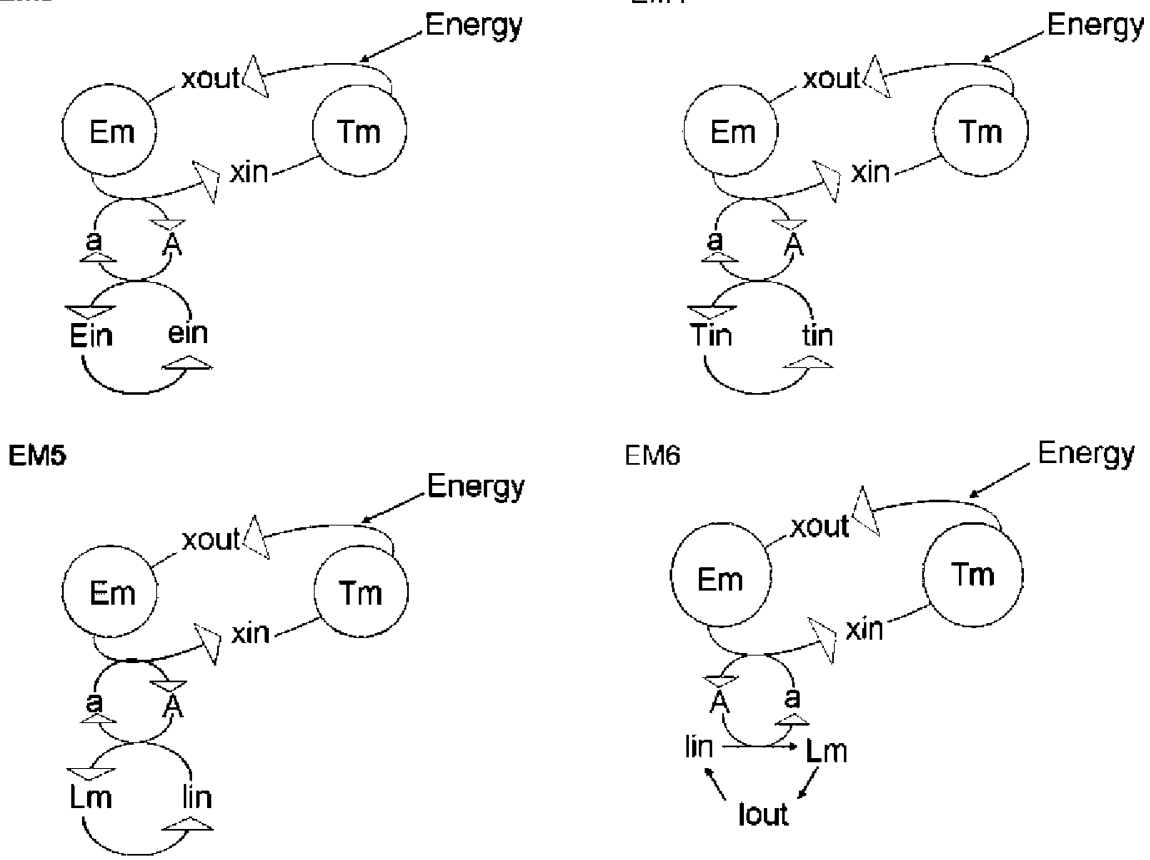

Fig. 2. Schematic representation of the Elementary Modes (EMs) described in Table 2.

for $L_{m}$ that is involved in the reactions of association/ dissociation to the membrane. The remaining four modes are all futile cycles that dissipate energy. Like the previous two, they also need the species $E_{m}$ and $T_{m}$ as catalysts.

\section{Enzyme-maintaining modes}

In order to take the dual character of $E_{m}, T_{m}$, and $L_{m}$, as both intermediates and catalysts, into account, and to have biochemically meaningful flux distributions, it is necessary to extend the stoichiometric modelling of metabolic networks as in the following definition:

Definition 1. A set of reactions that can operate at steady state is said to be an enzyme-maintaining mode if it produces its own catalysts.

Thus, an enzyme-maintaining mode of the metabolism of a self-maintaining proto-cellular system must contain the reactions to form the compounds that catalyze its own reactions. This is the only way to ensure its stationary functionality when the catalytic compounds of the reaction network are subject to degradation.

In general, EMs are not enzyme-maintaining modes. EMs must be combined adequately to find an enzymemaintaining mode. Eventually, the suitable combination of EMs can give rise to an enzyme-maintaining mode. However, these enzyme-maintaining modes are not necessarily elementary since the removal of any reaction of the network does not necessarily prevent a steady flux. So, a new minimalist condition must be imposed.

According to EM analysis, elementarity for enzymemaintaining modes could be defined according to the minimality in the number of reactions required to work stoichiometrically at steady state. However, the implementation of only this condition results insufficient since some reaction of the system might not appear in any of the resulting enzyme-maintaining modes. To avoid this possibility, the following condition of elementarity is imposed: every reaction involved in the EMs must appear in at least one enzyme-maintaining mode. Consequently, for this kind 
of enzyme-maintaining systems, the concept of elementarity must referred to each reaction of the network.

Definition 2. An enzyme-maintaining mode is elementary with respect to a given reaction if the inhibition (removal) of any of the remaining reactions causes the cessation of any steady state flux through the reference reaction.

Notice that under this definition all the EMs are represented in the set of elementary enzyme-maintaining modes. Since an elementary enzyme-maintaining mode is not always elementary with respect to all the reactions, as occurs in the example analyzed below, an elementary enzyme-maintaining mode with respect to a given reaction could contain other elementary enzyme-maintaining modes with respect to different reactions.

Since EMs are the basic stoichiometric units of the metabolic network, they can be used as blocks to build up all the elementary enzyme-maintaining modes. A standard procedure can be summarized as follows:

1. For each reaction of the metabolic network determine the EMs in which this reaction is involved.

2. Check if any of these EMs is an enzyme-maintaining mode.

3. The process is finished for the EMs that are enzymemaintaining modes.

4. Combine the remaining EMs that are not enzymemaintaining modes with the other EMs until an elementary enzyme-maintaining mode is formed.

5. Proceed in this way until all the reactions of the network have been analyzed.

Let us consider the EMs obtained in the previous section. Both EMs 1 and 2 require $E_{m}, T_{m}$, and $L_{m}$ but these compounds are not produced by them. Therefore, neither EMs 1 and 2 are enzyme-maintaining modes. A similar reasoning proves that none of the remaining EMs presented in Table 2 are enzyme-maintaining. However, the combination of them gives rise to the full set of elementary enzyme-maintaining modes $\left(Z M_{i}\right)$ of the selfmaintained proto-cellular system:

- $Z M_{1}=\lambda_{11} E M_{1}+\lambda_{21} E M_{2}+\lambda_{51} E M_{5}$,

- $Z M_{2}=\lambda_{12} E M_{1}+\lambda_{22} E M_{2}+\lambda_{62} E M_{6}$,

- $Z M_{3}=\lambda_{13} E M_{1}+\lambda_{23} E M_{2}+\lambda_{43} E M_{4}+\lambda_{53} E M_{5}$,

- $Z M_{4}=\lambda_{14} E M_{1}+\lambda_{24} E M_{2}+\lambda_{44} E M_{4}+\lambda_{64} E M_{6}$,

- $Z M_{5}=\lambda_{15} E M_{1}+\lambda_{25} E M_{2}+\lambda_{35} E M_{3}+\lambda_{55} E M_{5}$,

- $Z M_{6}=\lambda_{16} E M_{1}+\lambda_{26} E M_{2}+\lambda_{36} E M_{3}+\lambda_{66} E M_{6}$.

where $\lambda_{i j}$ are strictly positive real numbers for all $i, j . Z M_{i}$ vectors are not completely defined as they are formed by stoichiometrically independent modes. Thus there are an infinite number of ways of stoichiometrically adjusting each of the elementary enzyme-maintaining modes. It must be stressed that the last four enzyme-maintaining modes $Z M_{3}, Z M_{4}, Z M_{5}$, and $Z M_{6}$ are elementary only with respect to one reaction: the degradation of $E_{i n}$, in the case of EMs 3 and 4, and the degradation of $T_{i n}$, in the case of modes 5 and 6 . On the other hand, it is easy to verify whether the first two, $Z M_{1}$ and $Z M_{2}$, are elementary with respect to all the reactions involved in the respective modes. Notice that EMs 1 and 2 are present in all elementary enzyme-maintaining modes because they are the sources of both enzymes $E_{m}$ and $T_{m}: E_{m}$ is produced in the EM 1, whereas $T_{m}$ is formed in the EM 2. The RSs remain the same as before. All the elementary enzyme-maintaining modes $Z M_{i}$ transform $e_{\text {out }}$ and $t_{\text {out }}$ into $T_{\text {out }}$ and $E_{\text {out }}$, although with a different consumption of energy; the first two need three units whereas the remaining requires four units of energy.

The solution space of the proposed self-maintained proto-cellular system can be generated from the six elementary enzyme-maintaining subnetworks. Formally, if RS is a generic vector of RSs, then

$$
\begin{aligned}
\mathbf{R S}= & \mu_{1} Z M_{1}+\mu_{2} Z M_{2}+\mu_{3} Z M_{3}+\mu_{4} Z M_{4} \\
& +\mu_{5} Z M_{5}+\mu_{6} Z M_{6}
\end{aligned}
$$

with $\mu_{i} \geqslant 0$ for all $i=1, \ldots, 6$.

It must be remarked that this set of vectors is not completely defined due to the indeterminacy of the generators $Z M_{i}$. Nonetheless, this general solution is contained in the whole convex solution space generated by the six EMs $\left(E M_{i}\right)$ (see Eq. (1)).

\section{Concluding remarks}

The definition and the very essence of a self-maintained proto-cellular system imply the inclusion of the enzyme compounds (or in general the catalyst elements) in the network model as metabolic intermediates: they need to be synthesized and degraded as a result of the actual network system. This condition was advanced by Robert Rosen in his ground-breaking paper presented in the late 1950s (Rosen, 1958a, b, 1959) and recently emphasized by Letelier et al. (2006) and by Cornish-Bowden and Cárdenas (2008). One immediate consequence of this dual condition of the enzymes in self-maintained proto-cellular systems is that the structural study of this type of metabolic networks needs to be modified. In this paper, the definition of elementary self-maintaining mode has been presented, which includes the restriction arising out of the double nature of some network intermediates: metabolic and catalytic. This approach goes further with a view to establishing the organizational characteristics of biological systems (see, for instance, Fontana and Buss, 1994; Dittrich and di Fenizio, 2007).

The application of this approximation to a previously proposed simple model of self-maintained proto-cellular system (Olasagasti et al., 2007) has allowed us to find all its elementary enzyme-maintaining modes, as well as a convex basis to generate all possible stoichiometric transformations. Contrary to the conventional-space basis, the new one is formed by undetermined modes, as they come from the coupling of the EMs. The formalization of this study as 
well as the extension to more complex metabolic systems and the elaboration of computational tools are under development and have provided us with directions for future research efforts.

\section{Acknowledgments}

This paper has been supported in part by Grants no. BFU2006-01951-BMC, FIS2006-08607 from MEC (Spain), and IMC PI-026/2007 (Tenerife, Spain).

We would also like to acknowledge the suggestions made by the two anonymous reviewers that helped us to attain this final version.

\section{References}

Cornish-Bowden, A., Cárdenas, M.L., 2008. Self-organization in the origin of life. J. Theor. Biol., this issue, doi:10.1016/j.jtbi.2007.07.035.

Covert, M.W., Palsson, B.O., 2003. Constrained-based models: regulation of gene expression reduces the steady-state solution space. J. Theor. Biol. 221, 309-325.

Dittrich, P., di Fenizio, P.S., 2007. Chemical organization theory. Bull. Math. Biol. 69, 1199-1231.

Fontana, W., Buss, L.W., 1994. The arrival of the fittest-toward a theory of biological organization. Bull. Math. Biol. 56, 1-64.

Heinrich, R., Schuster, S., 1996. The Regulation of Cellular Systems. Chapman \& Hall, New York.

Kamp, A., Schuster, S., 2006. METATOOL 5.0: fast and flexible elementary modes analysis. Bioinformatics 22, 1930-1931.

Klamt, S., Stelling, J., 2006. Stoichiometric and constraint-based modelling. In: Szallasi, Z., Stelling, S., Periwal, V. (Eds.), System Modeling in Cellular Biology: From Concepts to Nuts and Bolts. MIT Press, Cambridge, pp. 73-96.

Letelier, J.C., Soto-Andrade, J., Guíñez Abarzúa, F., Cornish-Bowden, A., Cárdenas, M.L., 2006. Organizational invariance and metabolic closure: analysis in terms of (M,R) systems. J. Theor. Biol. 238, 949-961.

Nuño, J.C., Sánchez-Valdenebro, I., Pérez-Iratxeta, C., Meléndez-Hevia, E., Montero, F., 1997. Network organization of monosaccharide interconversion. Biochem. J. 324, 103-111.

Olasagasti, F., Moreno, A., Peretó, J., Morán, F., 2007. Energetically plausible model of a self-maintaining protocellular system. Bull. Math. Biol. 69 (4), 1423-1445.

Pfeiffer, T., Sánchez-Valdenebro, I., Nuño, J.C., Montero, F., Schuster, S., 1999. METATOOL: for studying metabolic networks. Bioinformatics $15,251-257$.

Rosen, R., 1958a. A relational theory of biological systems. Bull. Math Biophys. 20, 245-316.

Rosen, R., 1958b. The representation of biological systems from standpoint of the theory of categories. Bull. Math. Biophys. 20, 317-341.

Rosen, R., 1959. A relational theory of biological systems II. Bull. Math. Biophys. 21, 109-128.

Schilling, C., Palsson, B.O., 1998. The underlying pathway structure of biochemical reaction networks. Proc. Natl Acad. Sci. USA 95, 4193-4198.

Schilling, C.H., Schuster, P., Palsson, B.O., Heinrich, R., 1999. Metabolic pathway analysis: basic concepts and scientific applications in the postgenomic era. Biotechnol. Prog. 15, 296-303.

Schuster, S., Dandekar, T., Fell, D., 1999. Detection of elementary flux modes in biochemical networks: a promising tool for pathway analysis and metabolic engineering. Trend. Biotechnol. 17, 53-60.

Schuster, S., Fell, D.A., Dandekar, T., 2000. A general definition of metabolic pathways useful for systematic organization and analysis of complex metabolic networks. Nat. Biotechnol. 18, 326-332.

Skulachev, V.P., 1992. The laws of cell energetic. Eur. J. Biochem. 208, 203-209.

Stephanopoulos, G., Nielsen, J.B., Aristodou, A., 1998. Metabolic Engineering: Principles and Methodologies. Academic Press, San Diego.

Varela, F., Maturana, H., Uribe, R., 1974. Autopoiesis: the organization of living systems, its characterization and a model. BioSystems 5, 187-196. 